\title{
iRE: A Semantic Network based Interactive Requirements Engineering Framework
}

\author{
Kushal Ahmed*, Lian Wen and Abdul Sattar \\ Institute for Integrated and Intelligent Systems, Griffith University, \\ 170 Kessels Rd, Nathan, QLD 4111, Australia \\ Email: kushal.ahmed@griffithuni.edu.au, \{1.wen, a.sattar\}@griffith.edu.au
}

\begin{abstract}
Requirements engineering (RE) involves processing informal natural language descriptions of system requirements into an integrated and structured formal specification. The traditional $\mathrm{RE}$ process is ad-hoc, laborious, time-consuming, and error-prone manual process. For large-scale software intensive systems, the RE process becomes very difficult to manage. It is further complicated by ill-defined, incomplete, and redundantly specified requirements. To streamline the process, an end-to-end seamless RE framework is therefore needed. In this paper, we present an overview of an end-to-end semi-automated changetolerant interactive requirements engineering (iRE) framework. It keeps and maintains the meta-level information of the interrelated requirements models into a semantic network model (SNM), and provides a method and processing system to derive an integrated and structured model. The iRE involves the requirements analysts in an interactive fashion during the modeling process.

Index Terms-Requirements Engineering, Behavior Engineering, Behavior Trees, Semantic Network Model, Defects Detection, Change Management, Model Integration.
\end{abstract}

\section{INTRODUCTION}

Requirements Engineering (RE) often deals with a set of scattered and unstructured system requirements with a view to derive an integrated and structured system model that would serve as a formal specification. The requirements are usually represented by graphical models. The requirements analyst (RA) manually visits every model along with its interrelated models, and perform sanity and integrity checking. In this process, the RA, temporarily or finally, makes decisions on which requirement models are at that moment ready to be part of the formal specification. This decision may affect decisions made for other interrelated models in a cascading fashion.

Nevertheless, the traditional RE process is ad-hoc, laborious, time-consuming, and error-prone manual process. It is further complicated by ill-defined, incomplete, and redundantly specified requirements. Sometimes new requirements may be introduced, while existing requirements may be modified or deleted. Since the requirements are interrelated, a change in a requirement affects the acceptability of its related requirements for inclusion in the specification; which may again have ripple or cascading effects on other requirements further. Putting all these together, the whole process becomes very difficult to manage for a large-scale software intensive system, even if it is somewhat manageable for a small-scale one. To streamline the process, an end-to-end RE framework is therefore needed.

In this paper, we present an overview of a new end-toend semi-automated change-tolerant interactive requirements engineering (iRE) framework. It keeps and maintains the meta-level information of the given requirements models and their interrelations into a semantic network model (SNM), and provides a method and processing system to derive an integrated and structured model. The iRE involves the RA in an interactive fashion in the whole modeling process. We also have developed a prototype GUI toolkit [1] for the behavior trees (BTs), a modeling language used in Behavior Engineering ([2]-[4]). BT notation can be used in the entire RE process. We have not chosen other modeling languages such as the Unified Modeling Language (UML) because they pose additional significant challenges due to their use of a number of different models (e.g. use cases, sequence diagrams, state charts, etc.) in the process, and thus making the formalisation of an end-to-end seamless RE framework difficult.

Paper organisation: Section II describes the background about RE process using BTs; Section III briefly describes our interactive RE framework; Section IV illustrates the iRE; Section V describes our GUI toolkit; Section VI explores related literature; and finally Section VII presents our conclusion.

\section{BACKGROUND}

Consider the requirements of the Security Alarm System (SAS) ([5]) shown in Table I. The SAS will be used as a running example in this paper.

1) SAS: The SAS when activated by pressing a set button displays its active status. While active, the SAS can detect motion and can then make alarm sound. The alarm sound can be deactivated by entering a 3-digit code, which can be cleared and re-entered, if a mistake is made. From these requirements, an integrated and structured model would be developed.

TABLE I

REQUIREMENTS OF THE SECURITY ALARM SYSTEM

\begin{tabular}{|l|l|}
\hline R1 & The detector of SAS sends a trip signal when motion is detected. \\
\hline R2 & The SAS is activated by pressing the Set button. \\
\hline R3 & The set button is illuminated when the SAS is set. \\
\hline R4 & $\begin{array}{l}\text { If a trip signal occurs while the SAS is set, a high-pitched tone } \\
\text { (alarm) is emitted. }\end{array}$ \\
\hline R5 & A three-digit code must be entered to turn off the alarm tone. \\
\hline R6 & Correct entry of the code deactivates the SAS. \\
\hline R7 & $\begin{array}{l}\text { If a mistake is made when entering the code, the user must press } \\
\text { the Clear button before the code can be re-entered. }\end{array}$ \\
\hline
\end{tabular}

Note that the SAS is not a representative of the scale of problems that we intend to deal with. The SAS is rather a carefully selected small problem that is interesting enough to elucidate our model and mechanisms. 
In this paper, we represent the SAS using BTs i.e. behavior trees. BTs come very close to natural language specification in terms of what they can express [6]. They capture dynamic behaviors of a system in an arguably easy-to-understand graphical format in a manner which stays close to the structure and terminology of the original requirements, but possess a formal semantics [4]. We argue BTs can be used seamlessly throughout the modeling process since it combines use cases, scenarios and state-based behaviors into a single model. Moreover, state machines can be derived from a behavior tree [7].

2) $S A S$ and BTs: Requirement R2 in Table I mentions the initialisation scenarios for activating the SAS. R2 is represented by a BT in Fig. 1(a) as BT2. Fig. 1(b) displays the full contents of a BT node. Each node is associated with (a) a component, which has a behavior described by (b) a behavior name and (c) a behavior type. The node has a tag consisting of (d) a status (e.g. original, implied $(+)$, and missing $(-)$ ) indicating the level of modifications made apart from the original requirements, and (e) a traceability link to trace the node back to the original requirements. It can also have (f) an operator and/or (g) label which describe the flow of control. The node further may have (h) related nodes to describe relational behavior with other components. A relational behavior may be described by the related component name, the type of relation (what, how, where etc) and its qualifier (on, at, in etc). Note the brackets show the syntaxes.

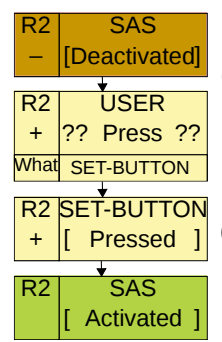

(e) traceability (a) component (f) node operator

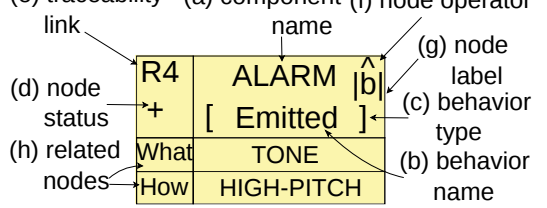

(a) BT2

(b) Attributes of a node

Fig. 1. Behavior Tree and Attributes of a node

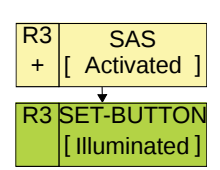

(a) BT3

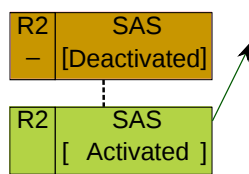

(b) Relation between BT2 \& BT3
Fig. 2. Behavior Trees (dots: nodes were skipped)

Behavior types are internal $(>. .<)$ and external $(\gg . . \ll)$ inputs, internal $(<. .>)$ and external $(\ll . . \gg)$ outputs, event (??..??), selection, (?..?), state realisation ([..]) etc (Fig. 3).

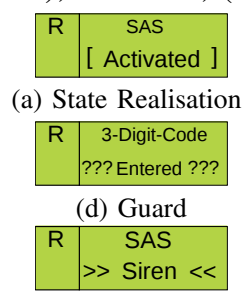

(e) External Input

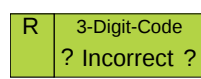

(b) Selection

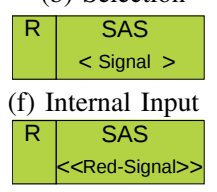

(f) External Output

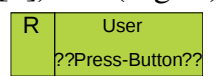

(c) Event

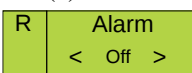

(i) Internal Output
Fig. 3. Behavior Types of a node.

The node operators are used to indicate control flow beyond simple sequential execution and branching. An operator (at source node) refers to another equivalent node (at target node) elsewhere in the behavior tree. The nodes of a behavior tree can be connected in different ways such as sequential, parallel, atomic and alternative ([3], [8]). Formal semantics have been provided to behavior trees using an extension of CSP which can capture state based information [4].

Notice BTs for Requirements R2 and R3 in Fig. 1(a) and 2(a). BT2's ending scenario activating the SAS is the starting scenario of BT3. This means, BT2 and BT3 are interrelated, and so the root of BT3 can be merged with the leaf of BT2 to obtain an integrated model. The integrated BT for BT2 and BT3 has been shown in Fig. 4(b). Informally, integration is performed by merging the associated nodes. Similarly, performing node merging between all BTs (the rest are in Fig. 4(c-g)), we can obtain an integrated holistic model that represents the whole SAS ([3], [8]). This process reveals inconsistencies, incompleteness, ambiguities and redundancies in the requirements ([3], [9]).

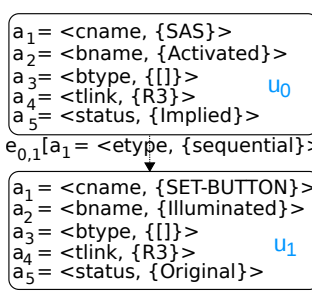

(a) BM (R3)

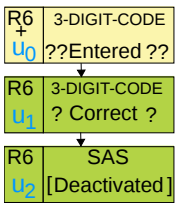

(d) BT6

R1 DETECTOR

$\mathrm{U}_{0}$ ??Detected??
What MOTION

R1 DETECTOR

$\mathrm{U}_{1}<$ Signal $>$

What TRIP

(e) BT1

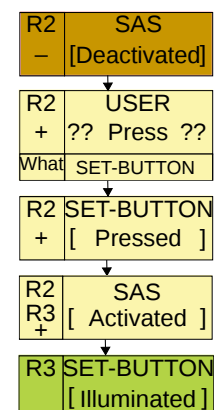

(b) BT2-3

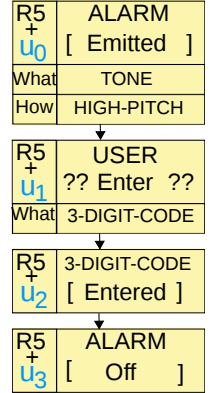

(c) BT5

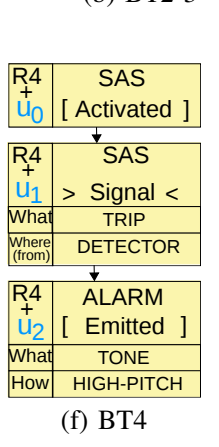

(f) BT4
Fig. 4. Mathematical Representation and Behavior Trees

3) Mathematical Representation of BTs: We use a very generic representation of Behavioral Model (BM), denoted by $m$, to refer to the mathematical representation of a BT [9]. As such, a BT node is referred to as Behavioral Unit (BU), denoted by $u$. A BU consists of a set of attributes $\left\{a_{k}\right\}$ and so does an edge between two BUs. Each attribute $a_{k}$ has a name, denoted by name $\left(a_{k}\right)$, and a value, denoted by value $\left(a_{k}\right)$. Fig. 4(a) shows a BM for BT3 having two BUs $u_{0}$ and $u_{1}$ connected by an edge $e_{0,1}$.

4) Equivalence Relation between BUs: We defined a similarity measure between two BUs $u$ and $u^{\prime}$ at [9]. The candidate BUs may not have the same set of attributes. Again, the values of the attributes may have compatibility issues. For example, state realisation and external/internal input/output 
are incompatible. We extend the definition to make the concept more generic. Let $A$ denotes the universal set of attributes; $A_{u} \subset A$ and $A_{u^{\prime}} \subset A$ denote the set of attributes of the BUs $u$ and $u^{\prime}$ respectively. Given that there exists no incompatible attribute pair in $A_{u}$ and $A_{u^{\prime}}$, the similarity measure $u \odot u^{\prime}=$ $\left(\sum_{i, j, k}\left(a_{i} \odot a_{j}\right) * w_{k}\right) / \sum_{k} w_{k}$ for all $a_{i} \in A_{u}, a_{j} \in A_{u^{\prime}}$ and $a_{k} \in A$ such that name $\left(a_{i}\right)=$ name $\left(a_{k}\right)=$ name $\left(a_{j}\right)$. Here, $a_{i} \odot a_{j}$ is the similarity of the attributes, and $w_{k}$ is the weight of $a_{k}$. For a given threshold $\alpha$, if $u \odot u^{\prime} \geq \alpha$, then $u$ and $u^{\prime}$ are equivalent. The weights and the threshold values may need to be configured locally for candidate BUs. For simplicity, lets assume a global configuration is used. Suppose the weights of component-name, behavior-name and behavior-type are 50\%, $25 \%$ and $25 \%$ respectively; all the rest are $0 \%$. Then the similarity between a BU $u_{2}$ of BT5 and the root BU $u_{0}$ of BT6 (Fig. 4(c, d)) becomes 0.75. If $\alpha=0.75$ then the two BUs are equivalent. Note that the weights and the threshold values may be estimated by AI techniques using the data from different case studies (future work). However, the RA may choose these values according to their experience. From our experience, the weight of the component-name should be higher than any other attributes for finding equivalence relations in BTs.

5) Integration Relation between BMs: A parent $\mathrm{BM} m_{p}$ forms an integration relation with a child $\mathrm{BM} m_{c}$, denoted by $R\left(m_{p}, m_{c}\right)$, if a $\mathrm{BU} u_{p}$ in $m_{p}$ is equivalent to the root $\mathrm{BU}$ $u_{c}$ of $m_{c}$. Notice BT5 and BT6 in Fig. 4(c, d). They form an integration relation because $u_{2}$ of BT5 and $u_{0}$ of BT6 are equivalent as shown above. The root BU of a child BM may be equivalent to multiple BUs of its parent BM. Thus, they may form multiple relations. We use $R^{k}\left(m_{p}, m_{c}\right)$ to denote $k$-th relation between $m_{p}$ and $m_{c}$. Depending on whether $u_{p}$ is the root, a branch or a leaf in the parent BM, we consider three types: root-root, branch-root and leaf-root relations [9]. Integration relations indicate a candidate merger of equivalent BUs and so a potential integration of two BMs into one.

\section{INTERACTIVE REQUIREMENTS ENGINEERING}

In a traditional RE process, natural language descriptions of a system's requirements are first translated by the RA into a given model, in our case behavioral models (i.e. BMs). The RA then manually visits every model along with its interrelated models, and perform sanity and integrity checking. The RA might temporarily or finally make decisions about whether a particular BM can be included in the specification or not. Once a decision has been made for a BM, normally the RA then finds another BM, interrelated to the former, to repeat similar kinds of checking and decision making.

In this process mentioned above, the RA might face a number of difficulties. For example, depending on the scale of the system, the RA might not have an overall knowledge on the requirements. So s/he might go through all the BMs and relations each time to find a suitable BM for his next consideration. Moreover, the RA has to remember previous decisions made for other BMs. Sometimes, the process could go back and forth, affecting previous decisions in a cascading fashion. With the absence of a clearly defined mechanism and any aid from an automated system, these issues must be addressed by the RA through laborious, time consuming and error-prone manual process. The whole process becomes even more complex for large-scale software intensive systems.

In this section, we provide an overview of a new framework of RE, referred to as Interactive Requirements Engineering (iRE). In iRE, the target integrated BM would be derived semi-automatically where the RA would get involved with the process in an interactive fashion. We do not opt for a fully automated system because explicit domain knowledge is required in various scenarios during the RE process.

The main concept of the iRE is to capture the metaknowledge about the BMs and their integration relations into a semantic network model (SNM). The iRE system (Fig. 5) would provide an interactive interface to the RA who could utilise the SNM to make domain decisions. A defects detection and resolution framework (DDRF) would detect potential defects [9] using the SNM and the interrelated BMs, and would suggest the RA a set of potential resolutions. The DDRF could prioritise the defects, and advise what's next to consider for making domain decisions. In the whole process, the RA may make changes in the BMs and/or their relations in the SNM. A change management framework (CMF) would synchronise the BMs and the SNM. The models integration framework (MIF) would automatically produce the integrated $\mathrm{BM}$ based on different configuration settings set by the RA.

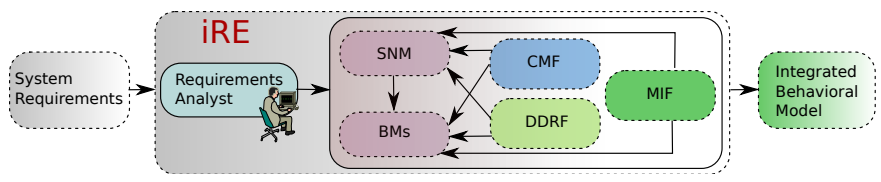

Fig. 5. Conceptual Model of iRE

Fig. 6 shows the process flow and Fig. 7 shows the elaborated view of the iRE.

When the RA translates the informal requirements into a set of BMs, the iRE process would determine the integration relations between the BMs (previous work [9]). The iRE then would build the SNM which is a graph with the BMs as its vertices and the integration relations between BMs as its edges. In the SNM, the RA may want to choose the initialisation $\mathrm{BM}(\mathrm{s})$. Note an initialisation BM consists of behavioral scenarios to initialise a system. The SNM then would undergo a sanity

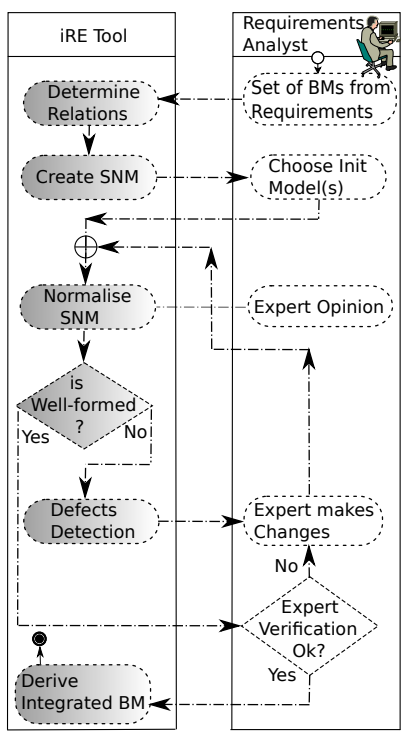

Fig. 6. Process flow of iRE checking, and decisions would be temporarily or finally made by the RA for its individual vertices i.e. the BMs to be ready for integration. Because of various types of relation in BMs, the temporary decisions could be revised back and forth, and 
thus could affect similar other decisions in a cascading fashion. In order to keep the cascading operation manageable, the SNM imposes a number of inference rules. Putting these altogether an SNM is called a normalised SNM when it passes the sanity checking and conforms to the inference rules. Expert opinion would be taken where necessary.

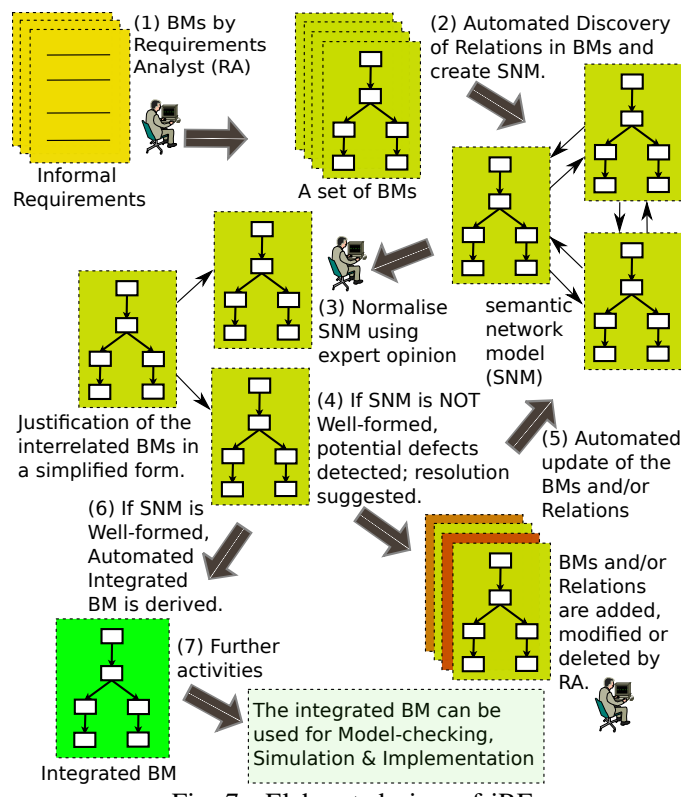

Fig. 7. Elaborated view of iRE

A normalised SNM is called a well-formed SNM when it is connected and it has one Init model. If the normalised SNM is not well-formed, the DDRF would detect potential defects; and would present the RA potential resolutions. The RA would make changes to the BMs and/or their interrelations in the SNM. The CMF would then reflect the changes and synchronise the BMs and the SNM with a view to get another normalised SNM from the previous normalised SNM. Once the SNM becomes well-formed and is verified by the RA, an integrated BM may be derived by the MIF (formalised in [10]). This integrated BM would be utilised to derive an executable specification, that is used for model-checking (e.g. [11]), simulation (e.g [12]) and further development works.

\section{ILLUSTRATION OF THE IRE FRAMEWORK}

In this section, we illustrate the functionalities of the components of the iRE framework using the BMs of the SAS.

\section{A. Determine Relations in the BMs.}

Let, $m_{1}$ to $m_{7}$ denote the BMs for $\mathrm{R} 1$ to $\mathrm{R} 7$ respectively. Using the same similarity measuring formula and parameters as in Section II, Table II shows the relations in them. Column $B M s$ shows parent and BMs (parent and child) having an integration relation, column Relations indicates the relation type, column $u_{p}$ shows the parent integration BU (note that the child integration $\mathrm{BU} u_{c}$ is omitted since it is always the root $\mathrm{BU}$ of the child $\mathrm{BM}$ ), and column $u_{p} \odot u_{c}$ shows the similarity measure between $u_{p}$ and $u_{c}$.
TABLE II

RELATIONS OF THE BMS OF THE REQUIREMENTS OF SAS.

\begin{tabular}{|c|c|c|c||c|c|c|c|}
\hline BMs & Relations & $u_{p}$ & $\odot$ & BMs & Relations & $u_{p}$ & $\odot$ \\
\hline$m_{2}, m_{3}$ & leaf-root & $u_{3}$ & 1 & $m_{2}, m_{4}$ & leaf-root & $u_{3}$ & 1 \\
\hline$m_{3}, m_{4}$ & root-root & $u_{0}$ & 1 & $m_{4}, m_{3}$ & root-root & $u_{0}$ & 1 \\
\hline$m_{4}, m_{5}$ & leaf-root & $u_{2}$ & 1 & $m_{6}, m_{2}$ & leaf-root & $u_{2}$ & 1 \\
\hline$m_{5}, m_{6}$ & branch-root & $u_{2}$ & .75 & $m_{5}, m_{7}$ & branch-root & $u_{2}$ & .75 \\
\hline$m_{6}, m_{7}$ & root-root & $u_{0}$ & 1 & $m_{7}, m_{6}$ & root-root & $u_{0}$ & 1 \\
\hline
\end{tabular}

\section{B. Develop SNM from the interrelated BMs.}

The SNM consists of vertices that represent the BMs and the edges that represent the integration relations between BMs. Fig. 8(a) shows a graph where the vertices refer to the BMs, and the edges refer to their relations annotated with similarity. We gradually develop the SNM as shown in Fig. 8(b).

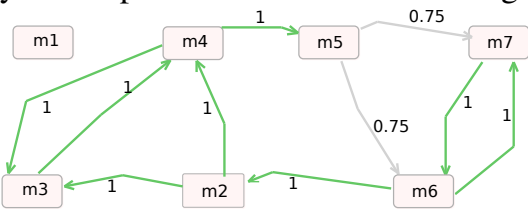

(a) Interrelated BMs, relations with similarity measure

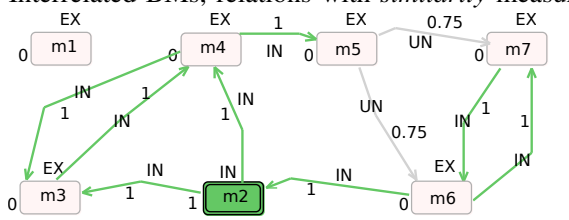

(b) SNM showing states, confidences and similarity measures Fig. 8. Interrelated BMs and SNM

Firstly, we annotate each vertex by assigning an acceptability state. We consider three states: Included (IN), Excluded (EX) and Undecided(UN). A BM is IN if the RA accepts it as valid, and thus the BM is included in the specification. A BM is EX if the RA does not accept it as valid and so is excluded from the specification, and it is UN if no decision has been made. Initially, all BMs are EX. To start, s/he selects a BM as an Init (initialisation) BM by discretion. Suppose s/he selects $m_{2}$ as the Init BM. We say it is Included in the specification and the CMF updates the SNM by assigning IN state to $m_{2}$ (Fig. 8(b), double border: Init).

We also annotate each vertex by assigning the confidence of each BM to quantify the credence for inclusion in the specification. We assign confidence 1 if a $\mathrm{BM}$ is $\mathrm{IN}$ and 0 if EX. Otherwise, confidence of a BM is the confidence of its parent multiplied by the similarity measure of its corresponding integration relation. For simplicity, we consider the maximum value when multiple parents exist.

In addition to the similarity measure, an integration relation is annotated by its state. We consider two layers of thresholds: (1) IN threshold $\alpha_{\text {IN }}$ and (2) UN threshold $\alpha_{\text {UN }}$ where $\alpha_{\mathrm{UN}}<$ $\alpha_{\mathrm{IN}}$. We assume a relation is IN if similarity $\geq \alpha_{\mathrm{IN}}$, and EX if similarity $<\alpha_{\mathrm{UN}}$, and UN otherwise. For domain dependency, the decision is committed on RA's consent.

\section{Develop Normalised SNM.}

After developing the SNM as shown in Fig. 8(b), the CMF derives a simplified version of the SNM where the relations are evaluated, minimised and organised, and the acceptability states and the confidences of the BMs are updated. 
Fig. 9(a) shows the root-root relations between $m_{3} \& m_{4}$. In this case, the integrated BM would be the same irrespective of which relation we consider. So, a root-root relation can safely be eliminated. From the candidate BMs, we choose a BM, referred to as the leader model, that is either an Init model or the closest model among others from an Init model. We then eliminate the relation incoming to that leader model. However, the elimination is committed upon the RA's consent. If there is a tie, the model having more (or equal) children is chosen as the leader model. In Fig. 8(b), $m_{4}$ is the leader among $\left\{m_{3}, m_{4}\right\}$. Even if their distance from the Init model $\left(m_{2}\right)$ is $1, m_{4}$ has more children (2) than $m_{3}$ (1). Similarly, $m_{6}$ is the leader among $\left\{m_{6}, m_{7}\right\}$. Therefore, the relations between $m_{3}$ $\& m_{4}$, and $m_{7} \& m_{6}$ are eliminated as shown in Fig. 9(b).

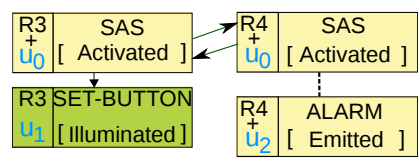

(a) root-root relations in $m_{3}$ and $m_{4}$

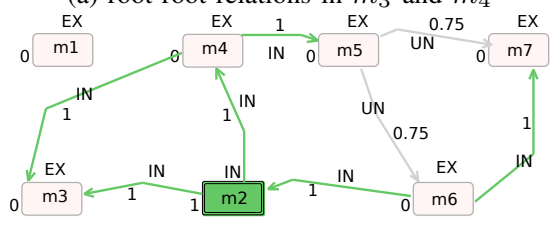

(b) root-root relations removed

Fig. 9. root-root relations

Again, Fig. 10(a) shows that $m_{2} \& m_{3}$, and $m_{2} \& m_{4}$ form leaf-root relations, and $m_{4} \& m_{3}$ form root-root relation. These three models will eventually be integrated into one model. Therefore, the root-root relation can safely be eliminated. The relation between $m_{6} \& m_{7}$ can also be eliminated. Fig. 10(b) shows the updated SNM.

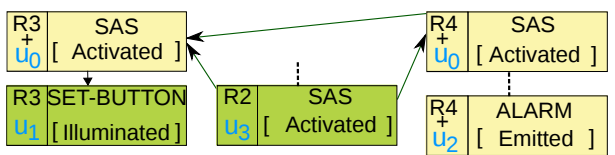

(a) transitive root-root relations in $m_{4}$ and $m_{3}$

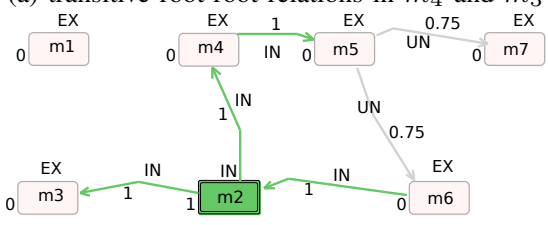

(b) transitive root-root relations removed

Fig. 10. Transitive root-root relations

A set of BMs may have root-root relations in a cyclic order, which can be simplified by choosing a leader model from them and create root-root relations from the leader model to all other models. It eliminates a relation as well. Again, the number of parent and child relations may change for a BM. So the leader model for a root-root relation may also change. In that case, the direction of the root-root relation can be reversed. Further, if a set of BMs is connected by root-root relations, they can be transformed into a tree-structure by selecting the leader and creating root-root relations from the Init model to all other BMs. It is similar to breaking cyclic root-root relations except it does not reduce the number of relations.
A BM can be validated before assigning an acceptability state. For example, the scenarios for the Init model $m_{2}$ can be examined to extract a set of rules:

1) (L1) if \{\} , then $\neg$ SAS-Activated. That is, by default SAS is not Activated.

2) (L2) if SET-BUTTON-Pressed, then SAS-Activated. This rule overrides rule $\mathrm{L} 1$.

The above rules have been expressed in Clausal Defeasible Logic (CDL) [13], which is implementable [14]. We can form a rule-set, from the above $\mathrm{L} 1$ and $\mathrm{L} 2$ rules i.e. rule-set $=$ $\{L 1, L 2\}$. Since incremental refinement of a (conflicting) rule set can be performed in non-monotonic logic ([15], [16]), the rule-set can be gradually developed from the BMs. In this paper, we assume that the BMs are valid against the rule-set.

Suppose the relation between $m_{5} \& m_{6}$ is accepted by the RA. As shown in Fig. 11(b), we can then infer IN states for $m_{3}, m_{4}, m_{5}$ and $m_{6}$. We further infer UN state for $m_{7}$ since neither its parent nor the relation is EX. Lastly, we infer EX for $m_{1}$ since it is no way connected with the Init model.

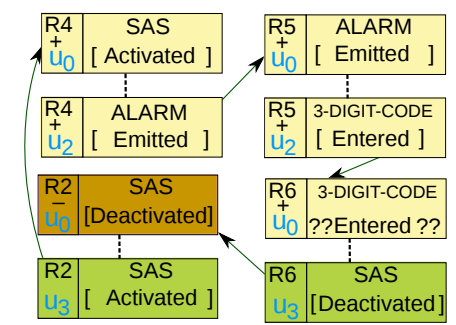

(a) Circular relation in BMs

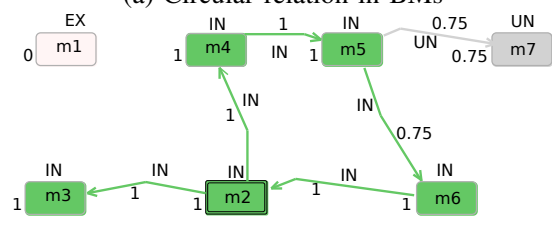

(b) Cycle in SNM

Fig. 11. Circular Relation in BMs and SNM

1) Inferring IN State: We set $\operatorname{IN}(m)$ if $m$ is an Init BM that the RA has chosen with discretion. We infer IN $(m)$ if it is valid against the rule-set and there exists at least one pair of IN state parent and relation.

2) Inferring UN State: We infer $\operatorname{UN}(m)$ if it is not IN, it is valid against the rule-set and there exists at least one pair of parent and relation such that both are not EX.

3) Inferring EX State: We infer $\operatorname{EX}(m)$ if it is neither IN nor UN.

Further, Fig. 11(a) shows a circular relation consisting of $m_{2}, m_{4}, m_{5}$ and $m_{6}$. Now, if these BMs are integrated, it over-specifies the scenarios of the behaviors. Fig. 12 (a) shows the integrated model where the border lines show the same sequence of behaviors $\left(\left\langle u_{0}, u_{1}, u_{2}, u_{3}\right\rangle\right.$ and $\left.\left\langle u_{10}, u_{11}, u_{12}, u_{13}\right\rangle\right)$. To avoid this over-specification, a relation must be eliminated. We again select $m_{2}$ as the leader model. Fig. 12(b) shows the updated SNM, which we refer to as normalised SNM.

\section{Defects Detection and Resolution}

The SNM aids finding out defects in the requirements earlier. The BM $m_{1}$ gets EX state and it does not have any relation, so requirement $\mathrm{R} 1$ is incomplete. Please refer to our 


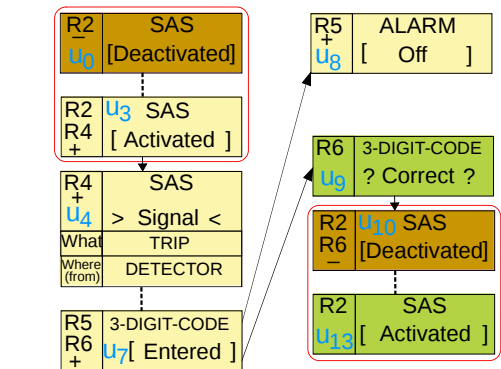

(a) over-specified integrated BM

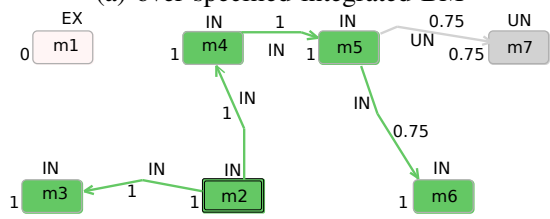

(b) Circular relation broken

Fig. 12. Over-specification (long arrows at (b) indicate branching)

previous paper [9] for techniques of potential defects detection. Defects may be resolved based on a set of resolution heuristics and suggestions could be presented to RA for making domain decisions, which are kept as future work.

\section{E. Develop Well-formed SNM}

This property determines whether the system requirements can be integrated into one formal specification or not. A normalised SNM is well-formed if (1) the number of Init models in the SNM is 1, (2) all models have IN states, and (3) all existing relations have IN states.

The RA may want to analyse why $m_{1}$ could not form a relation. The BM $m_{1}$ is about sending the trip signal when a motion is detected. It is easily understood that a precondition is missing. Suppose RA thinks that $m_{1}$ should happen when the SAS is activated (Fig. 13). Now, it forms root-root relations (similarity 1) with $m_{3}$ and $m_{4}$, and leaf-root relation

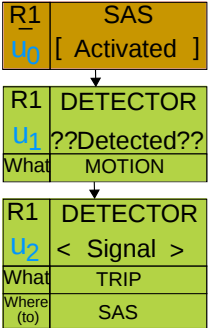

Fig. 13. Modified $m_{1}$ with $m_{2}$ (Fig. 14(a)). Fig. 14(b) shows the normalised SNM.

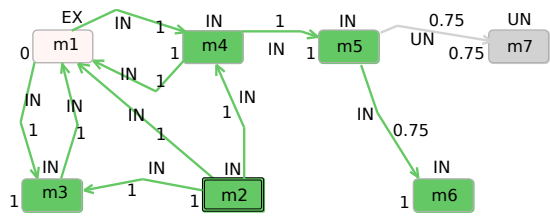

(a) SNM after modifying $m_{1}$

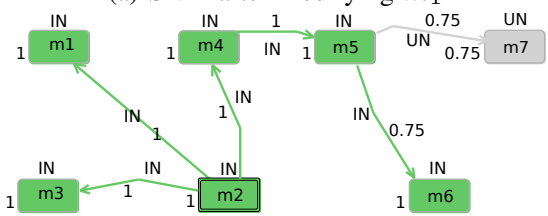

(b) normalised SNM after modifying $m_{1}$

Fig. 14. Modification of the BM $m_{1}$

If the RA accepts the relation between $m_{5}$ and $m_{7}$, the BM $m_{7}$ becomes IN. Fig. 15 shows the updated SNM, which is well-formed as well.

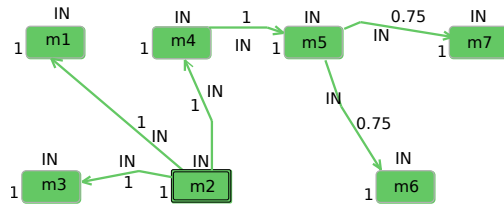

Fig. 15. well-formed SNM

\section{F. Derive Integrated Model}

The well-formed SNM can be traversed from the Init model utilising breadth-first-search, so an integrated BM can be derived as well. Fig. 16 shows the integrated BM of the SAS. The integration of BMs has been formalised at [10].

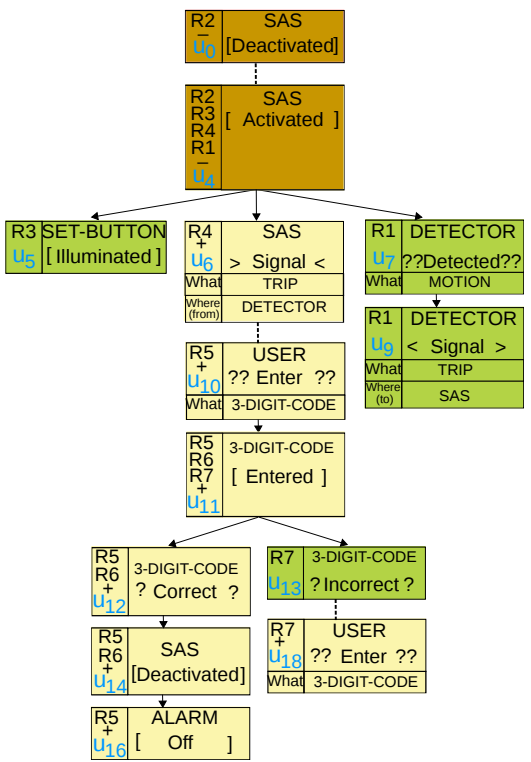

Fig. 16. Integrated BM for the SAS

\section{INTERACTIVE RE TOOL}

A GUI toolkit [1] has been developed using Eclipse Modeling Framework (EMF) and Graphical Modeling Framework (GMF). This tool demonstrates creating SNM from models and their relations. The link provided in [1] instructs how to install the tool. Many textual and graphical editors are available for behavior trees (e.g. [17], [18]). Among those, Oskar et al. [17] used the same underlying framework (EMF and GMF). Therefore, we plan to integrate the iRE with their editor.

\section{RElated WORK}

Traditional Behavior Engineering [3] provides an end-to-end process to derive a formal specification from a set of system requirements. It builds up a holistic view of the system by gluing the requirements together as if the RA is solving a jigsaw-puzzle. As a result, the process is ad-hoc and deriving a formal specification for a large-scale system becomes a laborious, time consuming and error-prone manual process. In this paper, we proposed a new end-to-end semi-automated changetolerant interactive mechanism for requirements engineering.

Kung et al. [19] performed impact analysis on class diagrams for a change in inheritance, encapsulation, polymorphism etc. Briand et al. ([20], [21]) derived an automated approach to analyse the change impact on different types of 
UML diagram such as Class Diagram, Sequence Diagram and State-chart Diagram. However, UML diagrams [22] represent variety of aspects of the system across several diagrams. Further, some UML diagrams do not have formal semantics. As these diagrams have different syntax and semantics, it often becomes difficult to define relationships between the diagrams. In UML, a number of overlapping partial views of the system along with the contextual switch when moving between diagrams makes it hard to formalise the impact of a change in the requirements in the modeling stage [23].

Göknil et al. [24] formally defined different types of relation in the requirements using first-order logic and developed change impact rules based on a requirement metamodel. However, they did not show how the requirement metamodel can be utilised for the management of the requirements models at earlier stage of system modeling. Wen and Dromey ([25]) proposed a traceability model to determine the impact of a change on the architecture and design of the system by embedding the changes in the specification behavior tree. To the best of our knowledge, there exists no framework that utilises a semantic network of behavioral models as knowledge-base in the earlier stage of requirements modeling.

Our proposed mechanism is highly inspired by the techniques applied to an epistemology-based system such as Ellis's belief system [26] from the Philosophy field and Doyle's Truth Maintenance System (TMS) [27] from the AI field. Ellis ascribes a sentence $A$ of a language $L$ as true $T$, false $F$ or an absence of firm belief $X$ and uses a number of acceptability criteria to determine the equilibrium (e.g. consistency, normalisation) condition. Doyle used a semantic network model as the epistemic state of belief, where the nodes refer to a set of objects of beliefs and links refer to the relations of the beliefs. Doyle also developed truth maintenance mechanisms to maintain the beliefs and the reasons of beliefs. In our model, justification of the acceptability of a BM depends on the validity against the rule-set and the related BMs. Our developed SNM also captures the confidence level of each of the BMs. Three types of states, along with the confidence level, explicitly portray a mental judgement on a BM.

\section{CONCLUSions AND Future WORK}

In this paper, we presented an overview of a new approach of requirements engineering. We used a semantic network model as a knowledge-base to capture the meta-level information of the system requirements and their relationships. We then illustrated how the SNM can support the requirements engineering in a seamless fashion. In near future, we would like to formalise each of the components (SNM, CMF, MIF and DDRF) of the iRE framework along with their implementation. We also plan to perform experimental evaluation of the iRE framework by emulating large-scale software intensive systems. Our plan also includes evaluating the approach using large-scale real case studies collaborating with industries.

\section{REFERENCES}

[1] "Interactive RE Demonstration Tool." [Online]. Available: (http://www.beworld.org/BE/ire/)
[2] R. G. Dromey, "Architecture As An Emergent Property of Requirements Integration," in ICSE, 2003, pp. 77-84.

[3] _ - "Formalizing the Transition from Requirements to Design," Mathematical Frameworks for Component Software-Models for Analysis and Synthesis, pp. 156-187, 2006.

[4] R. J. Colvin and I. J. Hayes, "A Semantics for Behavior Trees Using CSP with Specification Commands," Science of Computer Programming, vol. 76, no. 10, pp. $891-914,2011$.

[5] S. J. Prowell, C. J. Trammell, R. C. Linger, and J. H. Poore, Cleanroom Software Engineering: Technology and Process. Boston, MA, USA: Addison-Wesley, 1999

[6] P. Lindsay, "Behavior Trees: From Systems Engineering to Software Engineering," in 8th IEEE International Conference on SEFM. IEEE, 2010, pp. 21-30.

[7] S.-K. Kim, T. Myers, M.-F. Wendland, and P. A. Lindsay, "Execution of Natural Language Requirements using State Machines Synthesised from Behavior Trees," JSS, vol. 85, no. 11, pp. 2652 - 2664, 2012.

[8] T. Myers, "The Foundations for a Scaleable Methodology for Systems Design," PhD Thesis, School of ICT, Griffith University, Australia, 2011.

[9] K. Ahmed, T. Myers, L. Wen, and A. Sattar, "Detecting Requirements Defects Utilizing A Mathematical Framework for Behavior Engineering," JSCSE, Advanced Academic Publisher, vol. 3, no. 3, pp. 187-198, 2013.

[10] K. Ahmed, M. A. H. Newton, L. Wen, and A. Sattar, "Formalisation of the Integration of Behavior Trees," in 29th International Conference on Automated Software Engineering (ASE), Sep. 2014.

[11] P. Lindsay, N. Yatapanage, and K. Winter, "Cut Set Analysis Using Behavior Trees and Model Checking," Formal Aspects of Computing, vol. 24, pp. 249-266.

[12] T. Myers, R. G. Dromey, and P. Fritzson, "Comodeling: From Requirements to An Integrated Software/Hardware Model," IEEE Computer, vol. 44, no. 4, pp. $62-70$, April 2011.

[13] D. Billington, "Propositional Clausal Defeasible Logic," Logics in Artificial Intelligence, Lecture Notes in Computer Science, vol. 5293, pp. 34-47, 2008.

[14] D. Billington and A. Rock, "Propositional Plausible Logic: Introduction and Implementation," Studia Logica, vol. 67, no. 2, pp. 243-269, 2001.

[15] D. Billington, V. Estivill-Castro, R. Hexel, and A. Rock, "NonMonotonic Reasoning for RE: State Diagrams driven by Plausible Logic," 5th ENASE, pp. 68-77, 2010.

[16] L. W. Chan, R. Hexel, and L. Wen, "Rule-based behaviour engineering: Integrated, intuitive formal rule modelling," in 22nd ASWEC, June 2013, pp. 20-29.

[17] O. Rest, G. Wachsmuth, J. Steel, J. S, and E. Visser, in Theory and Practice of Model Transformations, ser. LNCS, K. Duddy and G. Kappel, Eds., 2013, vol. 7909.

[18] T. Myers, "TextBE: A Textual Editor for Behavior Engineering," 3rd Improving Systems and Software Engineering Conference (ISSEC), 2-5 August 2011.

[19] D. Kung, J. Gao, P. Hsia, F. Wen, Y. Toyoshima, and C. Chen, "Change Impact Identification in OO Software Maintenance," in ICSM, 1994, pp. 202-211.

[20] L. Briand, Y. Labiche, and L. O'Sullivan, "Impact Analysis and Change Management of UML Models," in ICSM, sept. 2003, pp. $256-265$.

[21] L. Briand, Y. Labiche, L. OSullivan, and M. Swka, "Automated Impact Analysis of UML Models," Journal of Systems and Software, vol. 79, no. 3, pp. $339-352,2006$.

[22] M. Chaudron, W. Heijstek, and A. Nugroho, "How Effective is UML Modeling?" SoSyM, vol. 11, pp. 571-580, 2012. [Online]. Available: http://dx.doi.org/10.1007/s10270-012-0278-4

[23] R. G. Dromey and D. Powell, "Early Requirement Defect Detection," TickIT Journal, pp. 3-13, 2005.

[24] A. Göknil, I. Kurtev, K. van den Berg, and J.-W. Veldhuis, "Semantics of Trace Relations in Requirements Models for Consistency Checking and Inferencing," SoSyM, vol. 10, pp. 31-54, 2011, 10.1007/s10270009-0142-3.

[25] L. Wen and R. G. Dromey, "From Requirements Change to Design Change: A Formal Path," in IEEE International Conference on SEFM, Beijing, 2004.

[26] B. Ellis, "Rational Belief Systems," Rowman and Littlefield, vol. 23, no. 1, pp. 109-114, 1979 .

[27] J. Doyle, "A Truth Maintenance System," AI, vol. 12, no. 3, pp. 231-272, 1979. 